\title{
Transgenic tobacco (Nicotiana tabacum L. cv. Samsun-NN) plants over-expressing a synthetic HRP-C gene are altered in growth, development and susceptibility to abiotic stress
}

\author{
Laura Heggie $^{\mathrm{a}, 1}$, Marcel A.K. Jansen ${ }^{\mathrm{b},{ }^{*}, 2}$, Emma M. Burbridge ${ }^{\mathrm{a}}$, Tony A. Kavanagh ${ }^{\mathrm{c}}$, \\ Roger N.F. Thorneley ${ }^{b}$, Philip J. Dix ${ }^{\text {a }}$ \\ ${ }^{a}$ Plant Cell Culture Unit, National University of Ireland, Maynooth, Co. Kildare, Ireland \\ b Department Biological Chemistry, John Innes Research Centre, Norwich NR4 7UH, UK \\ c Smurfit Institute of Genetics, Trinity College, University of Dublin, Ireland
}

Received 18 June 2005

Available online 05 December 2005

\begin{abstract}
The physiological role of class III peroxidases (EC 1.11.1.7) in controlling plant growth and development has been investigated by overexpression of both native and heterologous peroxidases. However, it has remained an enigma as to why the phenotypes of different peroxidase over-expressing transgenics vary. In order to resolve the conflicting information about the consequences of peroxidase over-expression, we have explored the role of the subcellular targeting of HRP-C in controlling stem growth, root development, axillary branching and abiotic stress tolerance in tobacco (Nicotiana tabacum L.). Altering the sub-cellular targeting of vacuolar HRP-C, such that over-expressed peroxidase accumulates in the cytoplasm and cell wall, induced phenotypic changes that are typically associated with altered auxin homeostasis, and overexpression of cell wall located peroxidases. We conclude that sub-cellular targeting is a determinant of the phenotype of peroxidase over-expressing plants.
\end{abstract}

(C) 2005 Elsevier SAS. All rights reserved.

Keywords: Peroxidase; Isozyme; Nicotiana tabacum; Lignin; Abiotic stress; Auxin

\section{Introduction}

Peroxidases are monomeric haem proteins that are ubiquitous in animals, plants and microorganisms where they are involved in both biosynthetic and degradative processes. Higher plants contain both ascorbate-oxidizing class I peroxidases and phenol-oxidizing class III peroxidases (EC 1.11.1.7). Class III peroxidases effect the one electron oxidization of aromatic phenols, amines, indoles, and sulfonates [31] using hydrogen per-

Abbreviations: HRP-C, horse radish peroxidase-C; IAA, indole-3-acetic acid; ROS, reactive oxygen species; TAP, tobacco anionic peroxidase; UV, ultraviolet.

${ }^{*}$ Corresponding author. Tel.: +353 214904558.

E-mail address: M.Jansen@UCC.IE (M.A.K. Jansen).

${ }^{1}$ Current address: School of Biological Sciences, University of Bristol, Woodland Road, Bristol, UK.

${ }^{2}$ Current address: Department of Plant Sciences (ZEPS), University College Cork, Cork, Ireland. oxide as the oxidant. The oxidation of phenolics leads to their cross linking to cell wall components or polymerization to form compounds such as lignin. The catalytic cycle of peroxidase normally involves three oxidation levels of the haem, the resting ferric state, the ferryl haem/porphorin radical cation state (Compound I) and the ferryl haem state (Compound II) [2]. An additional state of the enzyme with a ferrous haem can react with dioxygen to form Compound III, which is a key intermediate in initiating the oxidative decarboxylation of indole-3-acetic acid (IAA) [6].

Peroxidases have been implicated in several physiological and biochemical processes in planta, including regulation of cell growth and expansion [18,13], development [19], lignification and suberization [21], anthocyanin metabolism [32], IAA catabolism [6] and ROS generation [13]. Peroxidases are also thought to play an important role in the responses of plants to both abiotic and biotic stress conditions [11,13]. With these diverse roles, it may be anticipated that alterations in plant de- 
velopmental pathways, and stress responses, will be correlated with both expression levels and targeting of peroxidases. Such correlations are complicated by the large numbers of peroxidase genes that have been identified in a range of plant species $[26,29,32,35]$, the expression of which is controlled by specific developmental and/or environmental signals [8,26,35]. Moreover, physiological control mechanisms affecting enzyme activity have been proposed that include calcium-mediated activation and de-activation reactions [22]. At present, the functional role of this high level of genetic and physiological redundancy is not clear. However, transgenic plants over- and under-expressing several individual peroxidases have been produced in order to evaluate their roles in plant development, stress response and lignification.

Morphological alterations in tobacco plants over-producing the native tobacco anionic peroxidase (TAP) include a reduction in plant height, chronic wilting of mature plants, diminished axillary branching, reduced root development, a decrease in leaf thickness and delayed flowering [19]. Similarly, tobacco plants constitutively over-expressing a barley defense-related peroxidase were characterized by growth retardation [17]. Wilting was observed in transgenic tomato plants over-expressing a tomato peroxidase. These plants also exhibited a reduction in both root development and leaf thickness [3]. Some of the consequences of peroxidase over-expression on plant morphology are thought to result from changes in IAA levels. The IAA-oxidase activity of peroxidases has been extensively investigated under in vitro conditions [6,7], while in planta IAA levels were found to be locally decreased in tobacco plants over-expressing a tobacco peroxidase [20]. However, it is not clear whether peroxidase-mediated changes in IAA metabolism occur in all transgenic peroxidase over-expressing plants. It should be noted that no alterations in plant phenotype were observed following the abolition of two highly anionic peroxidases in tomato [28], the over-expression of a cucumber peroxidase in potato [25] and the over-expression of two sweet potato peroxidases in tobacco [9]. In some studies, the phenotype of the peroxidase over-expressing plant was found to be markedly different from that of the TAP over-expressing tobacco lines. HRP-Cla over-expressing tobacco and aspen plants were found to be fast growing. After 3 months of growth, the average stem length of some transgenic lines was $20 \%$ greater than that of the wild type [14]. No effects were observed on axial shoot or lateral root development $[14$, 15]. Thus, in these plants there is no phenotypic evidence for alterations in the physiologically relevant ratios of auxin to cytokinin [15]. Rather, it is proposed that over-expression of HRP$\mathrm{C} 1 \mathrm{a}$ induces alterations in the ascorbate/monodehydroascorbate ratio, which is directly relevant for the control of plant cell division and cell wall elongation $[1,12]$.

The results obtained to date with transgenic plants indicate that the relationship(s) between peroxidase over-expression and growth and development is complex. Although intuitively, peroxidase targeting should at least in part define the relationship between over-expression and growth, there is currently little experimental evidence to support this assertion. The sub-cellular expression of peroxidases has been determined in very few peroxidase over-expression studies. Wall-targeting of the re- combinant protein is often predicted, but is rarely experimentally confirmed. Over-expressed TAP was found to be localized in the cell wall of tobacco, an environment conducive to peroxidase activity and in which IAA is present [19]. Such localization is consistent with the measured changes in auxin levels as well as linked alterations in growth and development [20]. In contrast, Kawaoka et al. [15] found that in aspen plants most of the over-expressed HRP-Cla was present in the vacuoles, with a smaller amount in the cytoplasm.

An important question is whether the distinct growth effects of HRP-Cla over-expression [14] vis á vis over-expression of TAP [19] are due to the specific structure-function characteristics of these isozymes or due to differences in sub-cellular localization, or a combination of both. To answer this question, we have investigated the consequences of altered cellular targeting of HRP-C, in relation to growth, lignin accumulation and stress susceptibility. We have analyzed the growth and development of a transgenic tobacco line (ROPN3) that accumulates high levels of a synthetic horseradish peroxidase $\mathrm{C}$. The protein encoded by this synthetic gene is identical to that of mature HRP-Cla, except for an N-terminal alanine which is replaced in the synthetic peptide by a methionine (possibly post-translationally removed), and a C-terminal leucine that is missing. The HRP-C construct used to transform the ROPN3 plants lacks the $\mathrm{C}$-terminal, putative vacuolar targeting signal $[4,34]$, and as a consequence the bulk of the recombinant protein is retained and is active in the cytoplasm [16] (in this earlier paper ROPN3 is referred to as ROPN1). However, ROPN3 also secretes some active, recombinant HRP-C into the cell wall leading to wall activity levels up to 3.5 times higher than in wild-type plants [16]. We found that the ROPN3 line has many characteristics in common with plants that over-express class III peroxidases in the cell wall, including alterations in lignin composition, axillary branching, root development and stress-specific tolerance. We conclude that sub-cellular targeting is a key determinant of the phenotype of peroxidase overexpressing plants.

\section{Results}

Tobacco plants transformed with a synthetic HRP-C gene lacking the $\mathrm{C}$-terminal targeting sequence are characterized by relatively high peroxidase activity in the cytoplasm [16]. The ROPN3 line was selected as being representative of several, independently created, transformants with similar phenotypes [16]. We raised both wild-type and ROPN3 plants in glasshouses, under ambient light conditions. Peroxidase activities were routinely checked by measuring the rate of guaiacol oxidation by soluble extracts. It was found that guaiacol oxidation rates were on average ca. 10-fold higher in the ROPN3-line compared to the wild type (data not shown).

Fig. 1 shows a comparison of the growth of tobacco plants that over-express a synthetic HRP-C gene (ROPN) with that of the corresponding wild-type 'Samsun' line. We found that throughout development the height of the ROPN tobacco plants was consistently greater than that of the wild-type controls. For example, there was a significant difference of $120 \mathrm{~mm}$ in the 
A

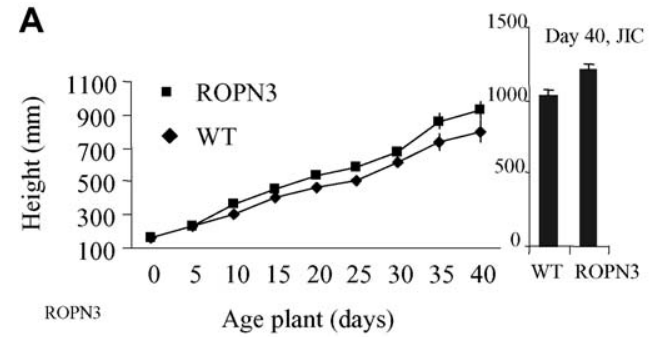

B

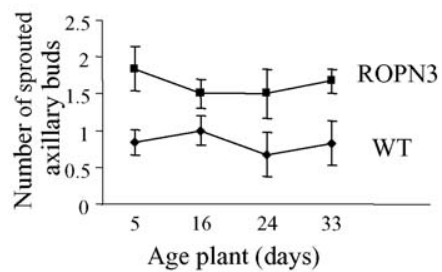

C

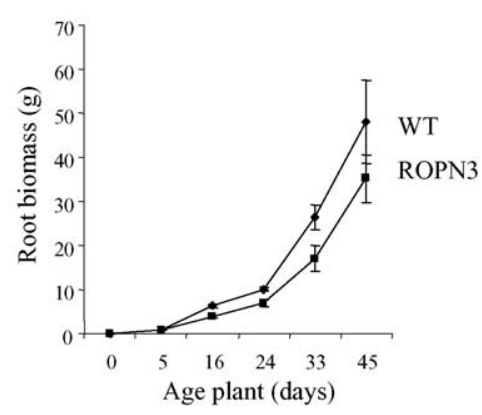

D

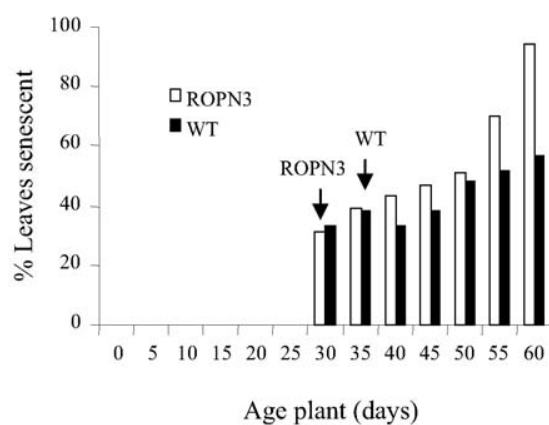

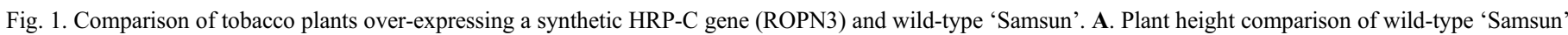

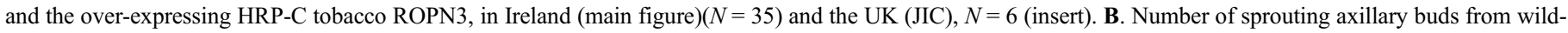

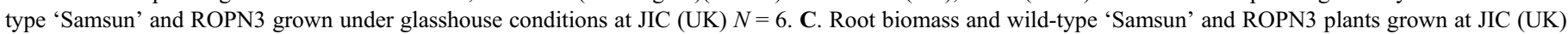

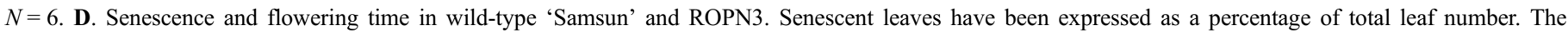

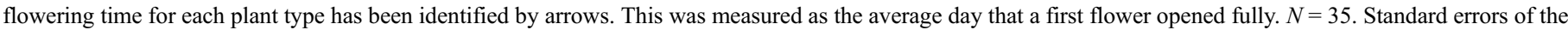
mean are shown in all graphs.

height of 35-day-old plants (Fig. 1A). At the end-point of the experiment (day 45), when plants had reached maturity (i.e. open flowers), there was a $100 \mathrm{~mm}$ difference in plant height (Fig. 1A). Growth and development can be quite variable depending on environmental parameters. However, the difference in height between the wild type and the ROPN3 transgenic is persistent, with similar differences being observed between the two lines whether the experiments were performed in Maynooth, Ireland (Fig. 1A line), or JIC, Norwich, UK. (Fig. 1A insert).

The increase in plant height in the ROPN3 line correlated with an increase in the number of leaf nodes. For example, the ROPN3 line had on average five more nodes on day 45 (Table 1). These data indicate an increase in the rate of cell division, rather than just cell elongation, in ROPN3 compared to the wild type. We found that the distance between two nodes was also greater in the ROPN3 line than in the wild-type 'Samsun'. For example, the distance between two nodes was, on the average, $10 \mathrm{~mm}$ longer in the ROPN3 line at day 45 (Table 1). Consistent with these observations, we found a significant increase in the biomass of the main stem in the ROPN3 line, although total shoot weight (main stem, plus branches, leaves and flowers), of which leaf weight is the main component, is not significantly different between the two lines (Table 1). Leaf and total plant biomass were also similar in the two tobacco lines. These data indicate that rather than an increase in primary productivity, the ROPN3 line differs from the wild type in terms of the re-distribution of resources.

Growth analysis showed that the ROPN3 plants developed significantly less root biomass than the wild type (Fig. 1C). This could already be observed in 2-3-day-old seedlings (data not shown). The differential in root biomass accumulation was maintained throughout development (Fig. 1C). ROPN3 tobacco plants had $13 \mathrm{~g}$ less root biomass than wild types by day 45 of the study. This is reflected in relatively low root/shoot ratios. For example, on day 45 the root/shoot ratios are 0.18 and 0.26 for the ROPN3 and wild-type lines, respectively (Table 1 and Fig. 1C).

The sprouting of axillary buds primarily typically reflects local cytokinin/auxin ratios. We found that the number of sprouting axillary buds was very limited in wild-type tobacco, under our growth conditions. However, a significant number of axillary buds, located on the main stem of the transgenic ROPN3 line, did sprout. The number of sprouting buds in-

Table 1

Morphological parameters in 45-day-old N. tabacum wild type and ROPN3

\begin{tabular}{|c|c|c|c|c|c|c|c|}
\hline & $\begin{array}{l}\text { Number } \\
\text { of nodes }\end{array}$ & $\begin{array}{l}\text { Internode } \\
\text { distance }\end{array}$ & $\begin{array}{l}\text { Shoot } \\
\text { biomass }\end{array}$ & $\begin{array}{l}\text { Stem } \\
\text { biomass }\end{array}$ & $\begin{array}{l}\text { Leaf } \\
\text { biomass }\end{array}$ & $\begin{array}{l}\text { Total plant } \\
\text { biomass }\end{array}$ & $\begin{array}{l}\text { Root/shoot } \\
\text { ratio }\end{array}$ \\
\hline Wild type Samsun NN & $21 \pm 2$ & $68 \pm 2 \mathrm{~mm}$ & $183 \pm 10 \mathrm{~g}$ & $76 \pm 6 \mathrm{~g}$ & $108 \pm 5 \mathrm{~g}$ & $228 \mathrm{~g}$ & 0.26 \\
\hline ROPN3 & $26 \pm 1$ & $78 \pm 1.5 \mathrm{~mm}$ & $200 \pm 8 \mathrm{~g}$ & $98 \pm 7 \mathrm{~g}$ & $107 \pm 4 \mathrm{~g}$ & $230 \mathrm{~g}$ & 0.18 \\
\hline
\end{tabular}

The errors represent the S.E. of the mean, $N=35$. 
creased approximately twofold in the HRP-C over-expressing line when compared with the wild type (Fig. 1B). Considering that cytokinin levels are most unlikely to increase in ROPN3 plants with impaired root development (Fig. 1C), these data are likely to reflect a decrease in auxin levels.

The development of the ROPN3 plants appeared to be faster than that of the corresponding wild-type line. ROPN3 plants flowered on average 10 days earlier than wild-type plants (Fig. 1D). However, there was no overall difference in flower number (data not shown). The onset of senescence was also earlier in the ROPN3 line (Fig. 1D). Thus, on day 60, some $95 \%$ of the leaves of the ROPN3 line had visually started to senesce. In comparison, only $58 \%$ of wild-type lines showed visual symptoms such as yellowing, wilting and necrosis.

A comparison of the phenolic contents in the ROPN3 and wild-type 'Samsun' lines was made since class III peroxidases show broad substrate specificities towards phenolic compounds. Plant phenolics can be grouped as bound and soluble compounds; together these determine, among others, the level of UV screening. We found a significant reduction in the amount of extractable lignin in the stems of ROPN3 tobacco plants (less than $1 \mathrm{mg}$ lignin/100 $\mathrm{mg}$ dried stem tissue) in comparison to wild-type 'Samsun' (3.5 mg lignin/100 mg dried stem tissue) (Fig. 2A). In a previous study [11], we showed that levels of soluble phenolic compounds are also dramatically decreased in the ROPN3 line (Fig. 2B). Together, these decreases in phenolic content may affect susceptibility of plants towards stresses. In contrast to the changes in lignin and soluble phenolics, chlorophyll contents were not significantly affected (data not shown). Yet, we noted small, but significant, changes in chlorophyll fluorescence values; both $F_{0}$ and $F_{\mathrm{m}}$ being slightly smaller in the ROPN3i line (Figs. 3A and C). The maximal efficiency of PSII did not alter in transgenic plants as compared to wild type (Figs. 3B and D), indicating that ROPN3 plants are not stressed as a direct result of the over-expression of the HRP-C gene, per sé. This observation is also consistent with the similar levels of biomass accumulation in these two lines.

The maximal efficiency of PSII $\left(F_{\mathrm{v}} / F_{\mathrm{m}}\right)$ is commonly used as a non-invasive indicator of damage to the photosynthetic machinery. Exposure of ROPN3 leaf discs of 25-day-old plants to broadband UV resulted in a strong decrease in PSII efficiency. The damaging effects of broadband UV radiation on $F_{\mathrm{v}} / F_{\mathrm{m}}$ are greater $(P>0.05)$ for the ROPN3 plants over-expressing HRP-
A

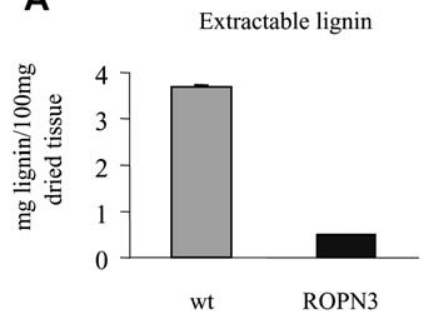

B

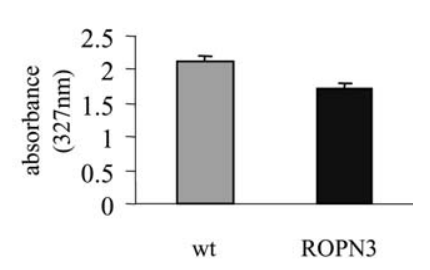

Fig. 2. Extractable lignin and soluble phenolic contents in wild-type 'Samsun' and ROPN3 plants. Error bars represent the S.E. of the mean, Lignin $N=3 \times 5$; Phenolics $N=10$. Student's $t$-tests indicate a significant difference $(P<0.01)$ between the two lines in terms of both parameters.
C, than for wild-type 'Samsun' plants (Figs. 3B and D). In wildtype plants, the decrease in $F_{\mathrm{v}} / F_{\mathrm{m}}$ is entirely due to a decrease in $F_{\mathrm{m}}$, indicating decreased PSII functionality (Fig. 3A). In ROPN3 plants there was an additional increase in $F_{0}$, reflecting supplementary dissociation of light harvesting pigments of the PSII core (Fig. 3C). Such dissociation can also be observed in wild-type plants that are exposed to more severe UV stress (data not shown). The increased sensitivity of ROPN3 is entirely consistent with the decreased levels of UV-absorbing phenolic polymers in this line (Fig. 2). It could be argued that the UV-sensitivity of the ROPN3 line reflects an overall sensitivity to stress. To investigate this possibility, we exposed both ROPN3 and wild-type plants to environmental stresses like excess photosynthetic radiation (photoinhibition), chilling or heat. These studies did not reveal any difference in the capabilities of the two lines to cope with these stresses [11].

\section{Discussion}

Tobacco plants over-expressing TAP in the cell wall are characterized by decreased axillary branching, decreased root development, increased lignification, and increased tolerance to UV stress (but not other abiotic stresses) [10,19]. This phenotype is clearly distinct from that of tobacco and aspen plants over-expressing HRP-Cla in the vacuoles which are characterized by increased stem elongation, increased tolerance to ROS, and unchanged lignin deposition $[14,15]$. We investigated whether sub-cellular targeting of class III peroxidases is a co-determinant of the phenotypic differences between transgenic lines. In the ROPN3 transgenes, the C-terminal vacuolar targeting signal of HRP-C has been removed, such that the bulk of the HRP-C is not secreted into the vacuole, but rather retained within the cell, with a smaller portion secreted into the cell wall domain [16]. We found that over-expression of peroxidase activity in ROPN3 resulted in poor root development and increased axillary branching (Table 2). These phenotypic effects might be caused by an increase in HRP-C activity per sé, and/or by altered subcellular targeting of this activity. Based on the comparison of ROPN3 with tobacco lines over-expressing the entire HRPC1a gene (Table 2) $[14,15]$, we conclude that the sub-cellular peroxidase distribution pattern is a determinant of plant phenotype. Tobacco plants accumulating high levels of TAP in the cell wall are characterized by altered root development and axillary branching [19], and this is attributed to subtle changes in auxin homeostasis [20]. Similarly, the ROPN3 line is altered in root development and axillary branching, and we interpret these data as reflecting a degree of cell wall targeted expression of ROPN3. This interpretation is consistent with the data presented by Kis et al. [16] who analyzed the sub-cellular distribution of peroxidase activity in the ROPN3 line and found significantly increased activity levels in the cellular domain.

It was found that the ROPN3 transgenic plants are relatively sensitive to broadband UV radiation. Previously, Jansen et al. [11] found that ROPN3 was also more sensitive to specific UVB wavelengths. Consistently, the ROPN3 line contains low levels of soluble phenolics and extractable lignin, which are both important UV-screening compounds. Tobacco plants over-ex- 
A
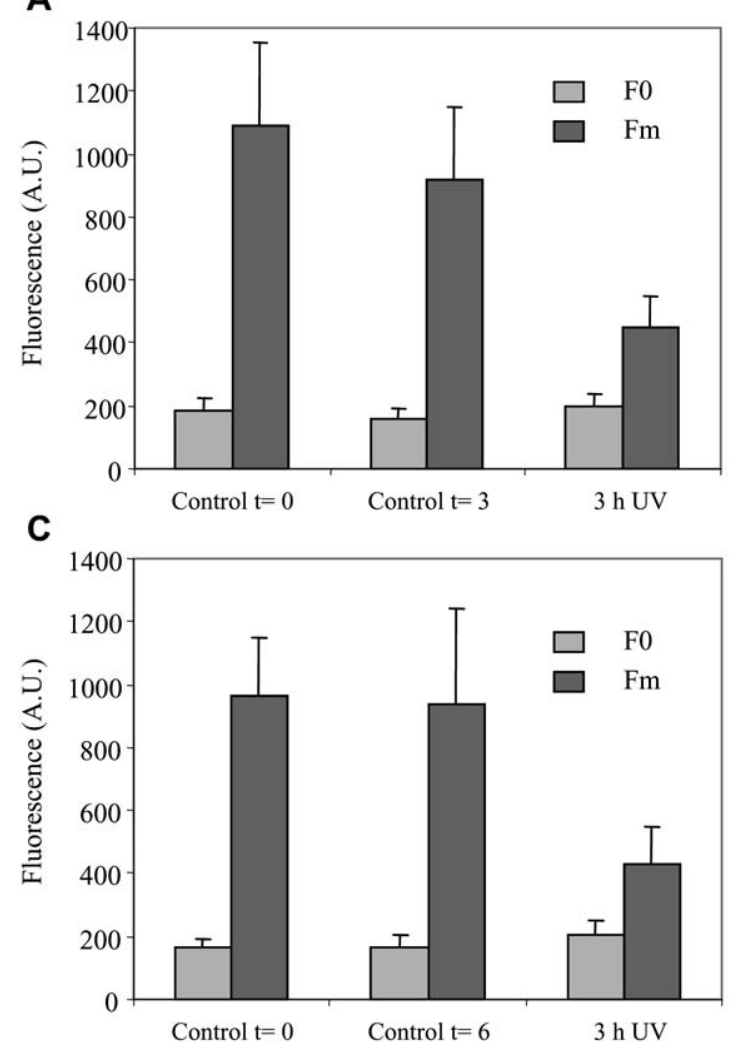

B

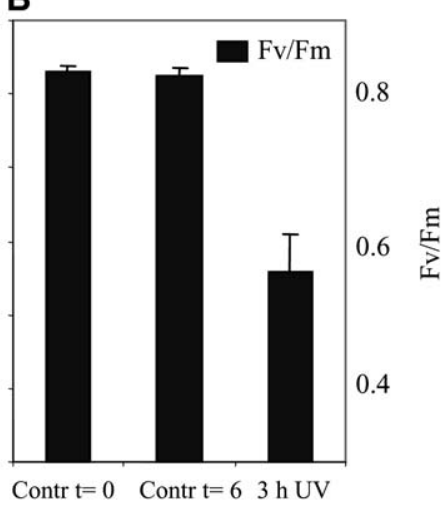

D

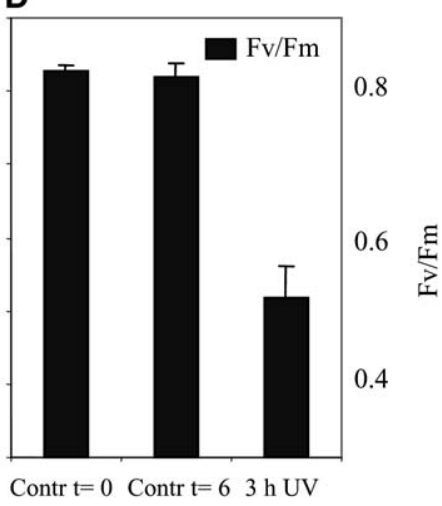

Fig. 3. Responses of plants to broadband UV radiation. Chlorophyll fluorescence parameters, including $F_{0}, F_{\mathrm{m}}$ and the efficiency of PS II $\left(F_{\mathrm{v}} / F_{\mathrm{m}}\right)$ were interpreted as a measure of plant health. (A) $F_{0}$ and $F_{\mathrm{m}}$ for wild type $(N=16-24) ;(\mathbf{B}) F_{\mathrm{v}} / F_{\mathrm{m}}$ for wild type $(N=16) ;(\mathbf{C}) F_{0}$ and $F_{\mathrm{m}}$ for ROPN3i $(N=16-24) ;(\mathbf{D}) F_{\mathrm{v}} / F_{\mathrm{m}}$ for ROPN3i $(N=16)$. Error bars represent the S.E. of the mean. Leaves were measured at the start of the experiment (Control $t=0)$; following 3 hours under a UVblocking filter (Control $t=3$ ) or following 3 hours under broadband UV. Student's $t$-tests indicated significant differences between wild type and ROPN3i for; $F_{0}$ and $F_{\mathrm{m}}$ (control $\left.t=0 ; P<0.05\right)$; and $F_{\mathrm{v}} / F_{\mathrm{m}}$ following UV treatment $(P<0.05)$.

Table 2

The impact of peroxidase over-expression in tobacco plants on phenotype and stress tolerance

\begin{tabular}{llll}
\hline & \multicolumn{2}{c}{ Genetically upregulated peroxidase } \\
\cline { 2 - 4 } & Tobacco anionic peroxidase ${ }^{\mathrm{a}}$ & $\begin{array}{l}\text { HRP-C lacking C-terminal } \\
\text { sequence (ROPN3) }{ }^{\mathrm{b}}\end{array}$ & HRP-C1a $^{\mathrm{c}}$ \\
\hline Length stem & Decreased & Increased & Increased $^{\mathrm{d}}$ \\
Root development & Decreased & Decreased & Unchanged \\
Root/shoot ratio & Decreased & Decreased & nd \\
Axillary branching & Decreased & Increased & Unchanged \\
Flowering & Late & Early & Early \\
Lignin deposition & Increased & Decreased & Unchanged \\
Abiotic stress tolerance & Specific, related to phenolics & Specific, related to phenolics & Non-specific increase ROS-scavenging \\
\hline
\end{tabular}

Final plant heights of flowering plants are similar for both wild-type and transgenic plants.

${ }^{a}$ Lagrimini et al. [19].

b This study.

c Kawaoka et al. [14,15].

${ }^{\mathrm{d}}$ Measured following 3 months of growth.

pressing TAP were found to be relatively UV-B tolerant [10], and this is related to increased levels of both soluble and lignin type phenolic polymers in these plants [19]. However, there is no general change in stress tolerance in these plants; both ROPN3 and tobacco over-expressing TAP [10] are unaltered in terms of their sensitivity towards three other abiotic stresses; photoinhibition, heat and chilling [11]. In contrast, plants overexpressing vacuolar HRP-C1a are tolerant to ROS which are key components of a broad range of abiotic and biotic stresses
[15]. Lignin deposition was unchanged in these plants. Based on these two distinct tolerance profiles, we consider that the altered stress-susceptibility of ROPN3 and TAP over-expressing lines is due to altered accumulation of UV-screening phenolics, while lines over-expressing vacuolar HRP-Cla have altered ROS scavenging abilities.

Thus, ROPN3 plants are characterized by alterations in four physiological parameters that are typically associated with expression of cell wall peroxidases and/or changes in auxin 
homeostasis [10,24]; altered lignin deposition, root development, axial branching, and specific stress tolerance. The observed increase in stem elongation and the early flowering can be explained by either alterations in ascorbate redox status $[14,15]$ or auxin homeostasis [27]. Based on these data, we conclude that by altering the sub-cellular distribution of vacuolar HRP-C, we have mimicked over-expression of a cell walllocated IAA-oxidizing peroxidase. However, the present data are significantly different to those of Lagrimini et al. [19]. We found increased rather than decreased, axillary branching and lignification, increased rather than decreased stem length and accelerated rather than delayed flowering in the transgenic ROPN3 line (Fig. 1 and Table 2). There are a number of possible explanations for these differences. Firstly, it is possible that the ROPN3 line retains some HRP-C vacuolar peroxidase activity, allowing features of vacuolar expression to be retained. Secondly, the kinetic characteristics and substrate specificities of TAP and HRP-C are distinctly different, and this includes the stability of compounds I, II and III, substrate binding and oxidation kinetics, and the reaction profile of IAA oxidative decarboxylation [5]. Thirdly, there is considerable variation in the physiological responses to changes in auxin levels. Local IAA concentrations and concentration gradients are important for many morphological responses [24], but are not always easily predicted in transgenic plant material where genes are constitutively expressed. Indeed, an inhibition of axillary sprouting and an increase in lignification have been linked with local decreases in IAA levels in tobacco plants over-expressing TAP [19], but also with major increases in IAA levels in tobacco with genetically increased IAA synthesis [27].

The complexity of the physiological responses to peroxidase over-expression is apparent. Our data clearly emphasize the importance of controlling sub-cellular targeting. We conclude that targeting of specific isozymes, together with variations in their substrate specificity and relative concentration, control the physiological response of the plant. Increased understanding of the regulation of expression levels, biochemistry and physiology of individual isozymes of the large peroxidase family is essential if their functionalities are to be exploited to improve agriculturally and ecologically important traits like plant growth, development, and stress tolerance.

\section{Methods}

\subsection{Plant material}

The generation of homozygous Nicotiana tabacum (L.) cv. "Samsun NN" transgenics over-expressing a synthetic horseradish peroxidase C (HRP-C) gene containing the natural Nterminal extension, but not the $\mathrm{C}$-terminal extension, and under control of the CaMV $35 \mathrm{~S}$ promoter, has been detailed previously [16] (in this previous paper ROPN3 was referred to as ROPN1). ROPN3 is one of several independent transgenic lines produced using the same ROPN construct encoding HRP$\mathrm{C}$ without its $\mathrm{C}$-terminal, putative vacuolar targeting signal. All lines had similar phenotypes, expression levels and sub-cellular distribution profiles.
Seeds of ROPN3 and control N. tabacum L. cv. 'Samsun'NN were germinated on MS salts [23] with $0.7 \%$ agar in the dark. Once the seedlings were established, they were sub-cultured onto fresh medium and maintained in a growth room $\left(25^{\circ} \mathrm{C}\right.$ with a $16 / 8 \mathrm{~h} \mathrm{light/dark}$ photoperiod) for 4 weeks before potting into peat-based compost. The plants were transferred to the glasshouse and growth analysis studies initiated after a 10day weaning-off period (day 0). Plants were monitored for changes in height, internode length, number of nodes, onset of flowering and flower number and the onset and number of senescent leaves over a period of 45 days.

\subsection{Peroxidase activity measurements}

Peroxidase activity was determined by measurement of the oxidation of guaiacol, as described in Kis et al. [16].

\subsection{Phenolic determinations}

A modified form of the method of Weiting et al. [33] was used to determine extractable lignin levels. Stem material from soil grown plants was ground in liquid nitrogen and extracted with methanol five times over 2 days. The insoluble material was dried at $60{ }^{\circ} \mathrm{C}$ overnight. The dried residue was incubated for 4 hours at $95{ }^{\circ} \mathrm{C}$ in $10 \%$ thioglycolic acid in $2 \mathrm{~N} \mathrm{HCl}$. After the pellet was washed with water, the ligninthioglycolic acid (LTGA) complex was resuspended in $0.5 \mathrm{M} \mathrm{NaOH}$ and incubated at $4{ }^{\circ} \mathrm{C}$ overnight. The sample was centrifuged and washed with water, and conc. $\mathrm{HCl}$ added to reprecipitate the LTGA complex. Once fully precipitated, the complex was collected and dissolved in $0.5 \mathrm{M} \mathrm{NaOH}$. The absorbance of the LTGA-NaOH complex was measured at $280 \mathrm{~nm}$ and the content of lignin calculated from a standard curve prepared with commercial alkali lignin.

The analysis of soluble, UV-absorbing pigments was based on extractions with acidic methanol. Leaf discs of equal size were incubated in a mixture of methanol, water and concentrated $37.5 \% \mathrm{HCl}$ (70\%:29\%:1\%) for at least 3 days at $4{ }^{\circ} \mathrm{C}$, in the dark. Absorbance of the extracts was measured spectrophotometrically [11].

\subsection{Stress treatments}

UV treatments consisted of exposure of tobacco leaf discs to UV radiation, generated by Philips TL12 fluorescent tubes $\left(\lambda_{\max } 315 \mathrm{~nm}\right)$. Leaf discs were exposed to $4.4 \mathrm{~W} / \mathrm{m}^{2}$ radiation for $3 \mathrm{~h}$. The irradiance level represents radiation in the spectral range between 280 and $345 \mathrm{~nm}$. Discs were floated on distilled water, with their adaxial side facing the UV source. The decrease in photosynthetic activity was attributed to the UV-B and UV-C wavelengths since the low level of UV-A radiation is ineffective in decreasing PSII activity. No additional PAR (photosynthetically active radiation) was applied during the UV treatment. UV levels were measured using an optometer (United Detector Technology Inc., Hawthorn, USA) equipped with a probe specific for UV wavelengths and/or a PMA2200 UV-meter (Solar Light Co., Philadelphia, USA). 
The severity of the stress effect was assessed by measuring the photosynthetic efficiency of PSII using the saturating pulse fluorescence technique (plant efficiency analyzer, Hansatech, King's Lynn, UK). The minimal fluorescence $\left(F_{0}\right)$, maximal fluorescence $\left(F_{\mathrm{m}}\right)$, and the variable fluorescence $\left(F_{\mathrm{v}}=F_{\mathrm{m}}-\right.$ $F_{0}$ ) were all measured according to van Kooten and Snel [30]. The photochemical yield of open PSII reaction centers, commonly known as the relative variable fluorescence, was calculated as $F_{\mathrm{v}} / F_{\mathrm{m}}$. It reflects the maximal efficiency of PSII which was measured in dark adapted tissue.

\section{Acknowledgements}

The authors appreciate advice by Dr. A.T. Smith, University of Sussex, Brighton, UK. The support of the European Commission through grant FRMX-CT98-0200, and of Enterprise Ireland through grant $\mathrm{SC} / 1997 / 104$, is gratefully acknowledged.

\section{References}

[1] O. Arrigoni, M.C. De Tullio, The role of ascorbic acid in cell metabolism: between gene-directed functions and unpredictable chemical reactions, J. of Plant Phys. 157 (2000) 481-488.

[2] H.B. Dunford, Heme Peroxidases, Wiley-VCH, New York, 1999.

[3] I. El Mansouri, J.A. Mercado, N. Santiago-Domenech, F. Pliego-Alfaro, V. Valpuesta, M.A. Quesada, Biochemical and phenotypical characterization of transgenic tomato plants over-expressing a basic peroxidase, Physiol. Plant. 106 (1999) 355-362.

[4] K. Fujiyama, H. Takemura, S. Shibayama, K. Kobayashi, J.-K. Choi, A. Shinmyo, M. Takano, H. Okada, Structure of the horseradish peroxidase isozyme C genes, Eur. J. Biochem. 173 (1988) 681-687.

[5] I.G. Gazaryan, L.M. Lagrimini, Purification and unusual kinetic properties of a tobacco anionic peroxidase, Phytochemistry 41 (1996) 10291034.

[6] I.G. Gazaryan, M. Lagrimini, G.A. Ashby, R.N.F. Thorneley, The mechanism of indole-3-acetic acid oxidation by plant peroxidases. Anaerobic stopped-flow spectrophotometric studies on horseradish and tobacco peroxidases, Biochem. J. 313 (1996) 841-847.

[7] I.G. Gazaryan, L.M. Lagrimini, F.A. Mellon, M.J. Naldrett, G.A. Ashby, R.N.F. Thorneley, Identification of the skatolyl hydroperoxide and its role in the peroxidase-catalysed oxidation of indol-3-yl acetic acid, Biochem. J. 333 (1998) 223-232.

[8] S. Hiraga, K. Sasaki, H. Ito, Y. Ohashi, H. Matsui, A large family of class III plant peroxidases, Plant Cell Physiol. 42 (2001) 462-468.

[9] G.-H. Huh, B.-W. Yun, H.-S. Lee, J.-K. Jo, S.-S. Kwak, Overproduction of sweet potato peroxidases in transgenic tobacco plants, Phytochem. 47 (1998) 695-700.

[10] M.A.K. Jansen, R.E. vandenNoort, M.Y.A. Tan, E. Prinsen, L. M. Lagrimini, R.N.F. Thorneley, Phenol-oxidizing peroxidases contribute to the protection of plants from ultraviolet radiation stress, Plant Physiol. 126 (2001) 1012-1023.

[11] M.A.K. Jansen, M. Elfstrand, L. Heggie, F. Sitbon, P.J. Dix, R.N. F. Thorneley, Over-expression of phenol-oxidising peroxidases alters the UV-susceptibility of transgenic Nicotiana tabacum, New Phytol. 163 (2004) 585-594.

[12] N. Kato, M. Esaka, Changes in ascorbate oxidase gene expression and ascorbate levels in cell division and cell elongation in tobacco cells, Physiol. Plant. 105 (1999) 321-329.

[13] T. Kawano, Roles of the reactive oxygen species-generating peroxidase reactions in plant defense and growth induction, Plant Cell Rep. 21 (2003) 829-837.
[14] A. Kawaoka, T. Kawamoto, H. Moriki, H. Ohta, M. Sekine, M. Takano, A. Shinmyo, Growth stimulation of tobacco plant introduced the horseradish peroxidase gene prxC1a, J. Ferment. Bioeng. 78 (1994) 49-53.

[15] A. Kawaoka, E. Matsunaga, S. Endo, S. Kondo, K. Yoshida, A. Shinmyo, H. Ebinuma, Ectopic expression of a horseradish peroxidase enhances growth rate and increases oxidative stress resistance in hybrid aspen, Plant Phys. 132 (2003) 1177-1185.

[16] M. Kis, E. Burbridge, I.W. Brock, L. Heggie, P.J. Dix, T.A. Kavanagh, An N-terminal peptide extension results in efficient expression, but not secretion, of a synthetic horseradish peroxidase gene in transgenic tobacco, Ann. Bot. (Lond.) 93 (2004) 303-310.

[17] B.K. Kristensen, J. Brandt, K. Bojsen, H. Thordal-Christensen, K. B. Kerby, D.B. Collinge, J.D. Mikkelsen, S.K. Rasmussen, Expression of a defence-related intercellular barley peroxidase in transgenic tobacco, Plant Sci. 122 (1997) 173-182.

[18] L.M. Lagrimini, Wound-induced deposition of polyphenols in transgenic plants overexpressing peroxidase, Plant Physiol. 96 (1991) 577-583.

[19] L.M. Lagrimini, V. Gingas, F. Finger, S. Rothstein, Y.L. Ting-Ting, Characterisation of antisense transformed plants deficient in the tobacco anionic peroxidase, Plant Phys. 114 (1997) 1187-1196.

[20] L.M. Lagrimini, The role of peroxidase in auxin metabolism. Abstracts Peroxidase '99. International Symposium for Plant Peroxidases, Columbus, Ohio, USA (1999).

[21] M. Lopez-Serrano, M.D. Fernandez, F. Pomar, M.A. Pedreno, A. R. Barcelo, Zinnia elegans uses the same peroxidase isoenzyme complement for cell wall lignification in both single-cell tracheary elements and xylem vessels, J. Exp. Bot. 55 (2004) 423-431.

[22] R. Medda, A. Padiglia, S. Longu, A. Bellelli, A. Arcovito, S. Cavallo, J. Z. Pedersen, G. Floris, Critical role of $\mathrm{Ca}^{2+}$ ions in the reaction mechanism of Euphorbia characias peroxidase, Biochemistry 42 (2003) 89098918.

[23] T. Murashige, F. Skoog, A revised medium for rapid growth and bioassays with tobacco tissue cultures, Physiol. Plant. 15 (1962) 473-497.

[24] J. Normanly, Auxin metabolism, Physiol. Plant. 100 (1997) 431-442.

[25] H. Ray, D.S. Douches, R. Hammerschmidt, Transformation of potato with cucumber peroxidase: expression and disease response, Physiol. Mol. Plant Pathol. 53 (1998) 93-103.

[26] K. Sasaki, T. Iwai, S. Hiraga, K. Kuroda, S. Seo, I. Mitsuhara, A. Miyasaka, M. Iwano, H. Ito, H. Matsui, Y. Ohashi, Ten rice peroxidases redundantly respond to multiple stresses including infection with rice blast fungus, Plant Cell Physiol. 45 (2004) 1442-1452.

[27] F. Sitbon, S. Hennion, B. Sundberg, C.H.A. Little, O. Olsson, G. Sandberg, Transgenic tobacco plants coexpressing the Agrobacterium tumefaciens iaaM and $i a a H$ genes display altered growth and indoleacetic acid metabolism, Plant Physiol. 99 (1992) 1062-1069.

[28] B.A. Sherf, A.M. Bajar, P. Kolattukudy, Abolition of an inducible highly anionic peroxidase activity in transgenic tomato, Plant Phys. 101 (1993) 201-208.

[29] M. Tognolli, C. Penel, H. Greppin, P. Simon, Analysis and expression of the class III peroxidase large gene family in Arabidopsis thaliana, Gene 288 (2002) 129-138.

[30] O. van Kooten, J.F.H. Snel, The use of chlorophyll fluorescence nomenclature in plant stress physiology, Photosynth. Res. 25 (1990) 147-150.

[31] N.C. Veitch, A.T. Smith, Horseradish peroxidase, Adv. Inorg. Chem. 51 (2001) 107-162.

[32] L.S. Wang, K. Burhenne, B.K. Kristensen, S.K. Rasmussen, Purification and cloning of a Chinese red radish peroxidase that metabolise pelargonidin and forms a gene family in Brassicaceae, Gene 343 (2004) 323335.

[33] N.I. Weiting, N.I. Paiva, R.A. Dixon, Reduced lignin in transgenic plants containing a caffeic acid $O$-methyltransferase antisense gene, Trans. Res. 3 (1994) 120-126.

[34] K.G. Welinder, Covalent structure of the glycoprotein horseradish peroxidase (EC1.11.1.7), FEBS Lett. 72 (1974) 19-23.

[35] K.G. Welinder, A.F. Justesen, I.V.H. Kjaersgard, R.B. Jensen, S. K. Rasmussen, H.M. Jespersen, L. Duroux, Structural diversity and transcription of class III peroxidases from Arabidopsis thaliana, Eur. Biochem. 269 (2002) 6063-6081. 